\title{
Electrical Switching in Thin Films of Nandi Flame Seed Cuticles
}

\author{
Wycliffe K. Kipnusu, ${ }^{1}$ Gabriel Katana, ${ }^{1}$ Charles M. Migwi, ${ }^{1}$ \\ I. V. S. Rathore, ${ }^{1}$ and Joshua R. Sangoro ${ }^{2}$ \\ ${ }^{1}$ Physics Department, Kenyatta University, P.O. Box 43844-00100, Nairobi, Kenya \\ ${ }^{2}$ Institute of Experimental Physics 1, University of Leipzig, 04103 Leipzig, Germany
}

Correspondence should be addressed to Wycliffe K. Kipnusu, wyckiprop@yahoo.com

Received 2 October 2008; Revised 13 January 2009; Accepted 19 January 2009

Recommended by Patric Jannasch

Current-voltage $(I-V)$ characteristics of Nandi flame seed cuticles (NFSCs) have been studied as a function of irradiation, annealing, and poling temperature. The cuticles showed memory and threshold switching. Threshold voltage $V_{\text {th }}$ was about $5 \mathrm{~V}$ which is almost five times higher than $V_{\text {th }}$ observed in synthetic polymers. The threshold voltage $V_{\text {th }}$ increased to 6-8 V after irradiation and annealing depending on the duration of annealing or irradiation. After switching, conductivity increased by an order of $10^{2}$. In reverse bias, increase of current was observed and the memory hysteresis loop was at higher conductivity than at the time of switching. Switching effect was minimized at a poling temperature of $370 \mathrm{~K}$. Formation of semiquinones and quinoid radicals from phenolic compounds may have contributed to electrical switching and hysteresis effect.

Copyright ( 2009 Wycliffe K. Kipnusu et al. This is an open access article distributed under the Creative Commons Attribution License, which permits unrestricted use, distribution, and reproduction in any medium, provided the original work is properly cited.

\section{Introduction}

Electrical switching and memory is the basis of many potential molecular electronic devices, and has gained considerable attention of numerous research efforts in recent years [13]. Aviram and Ratner [4] described the first concrete idea of molecular conductance switching in which the chemical structure consisted of an electron donating molecule and a modified acceptor connected via a saturated (insulating) $\sigma$ bridge. According to their theoretical calculations, insulating molecule becomes conductive after applying a certain threshold voltage that brings the $\pi$-levels in resonance. A range of other molecular structures which exhibit electrical threshold switching have been reported [1] including selfassembled monolayers of phenylethynyl oligomers [5] and rotaxanes oriented between two conductors by LangmuirBlodgett assembly $[6,7]$. Conductance switching has also been reported in synthetic polymeric materials which consist of large assemblies of molecules [8-12]. Whereas several biopolymers have well-documented properties as organic semiconductors [13-19], limited studies on electrical switching phenomenon of these biological assemblies have been done $[20,21]$.
Biopolymers are a rich resource for development of novel electronic devices, since there is tremendous natural variation in their composition [22]. Biopolymers also have the advantage of being biodegradable and biocompatible [23]. Plant seed cuticles have been shown to contain cutin and suberin biopolymers $[24,25]$. Cutin is the main component (between $40 \%$ and $85 \%$, w/w) of plant cuticles [26, 27]. Predominant aliphatic monomers of most cutins are $\mathrm{C}_{16}$ and $\mathrm{C}_{18} \omega$-hydroxylated fatty acids, typically carrying in addition hydroxy- or epoxy groups in midchain positions. Besides aliphatic monomers, cutin also contains glycerol and small amounts of phenolic compounds [28, 29]. Suberin occurs at abscission zones such as chalazal region of seed coats formed during late stages of seed maturation [30, 31]. Suberin contains both aliphatic and aromatic monomers $[32,33]$. The aliphatic part of suberin is mainly composed of $\mathrm{C}_{16}$ to $\mathrm{C}_{28} \omega$-hydroxy fatty acids and $\mathrm{C}_{16}$ to $\mathrm{C}_{26} \alpha, \omega$ dioic acids. The aromatic network is a hydroxycinnamatederived polymer, primarily comprised of ferulic acid, Nferuloyltyramine, cinnamic acid, p-coumaric acid, or caffeic acid [33]. Kipnusu et al. [34] have reported that Nandi flame seed cuticle is an insoluble biomaterial which has high-specific heats and shows glass transition temperature 


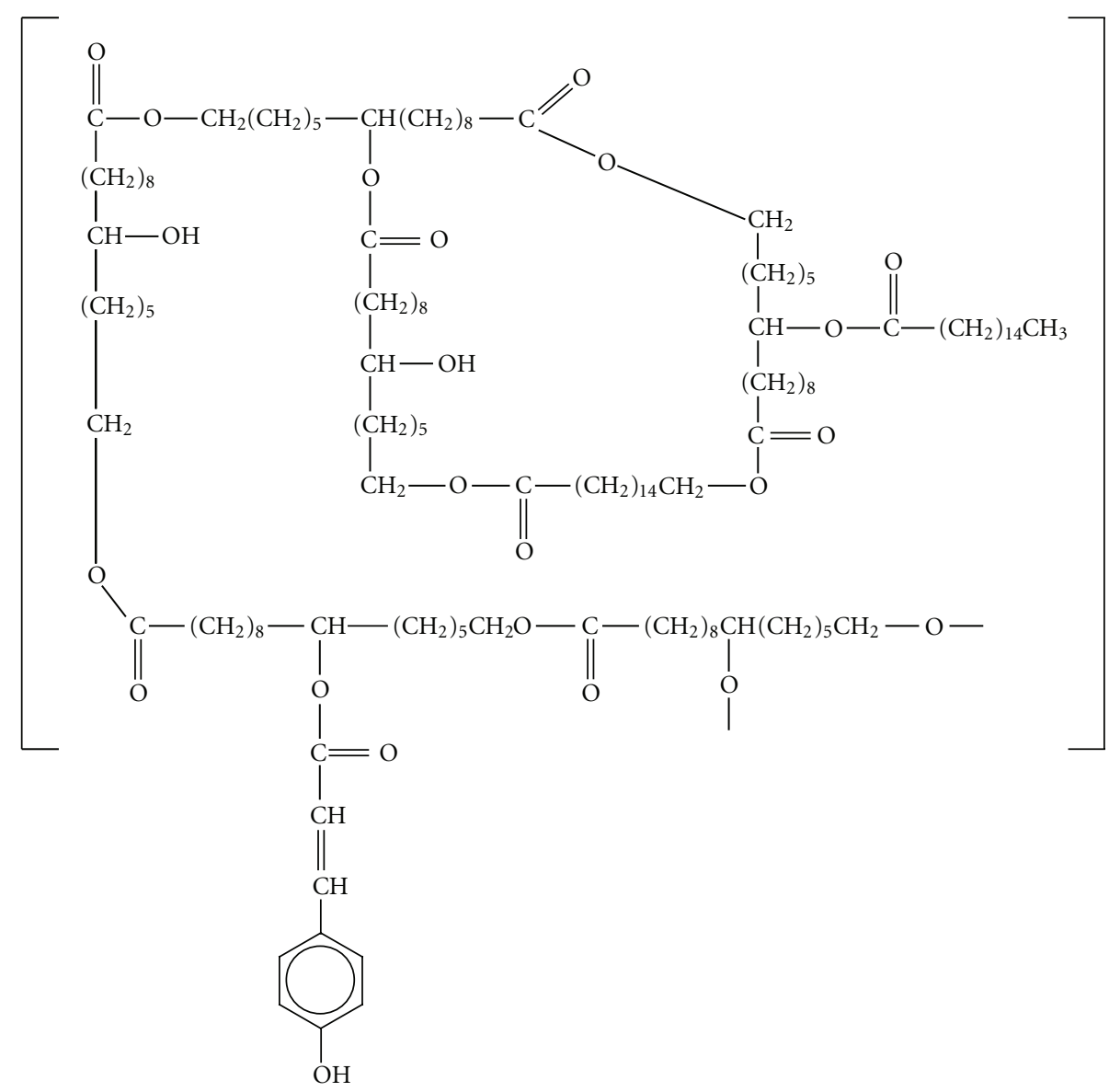

FIGURE 1: Models showing type of structures present in cutin biopolyester.

comparable to glass transition of cutin reported elsewhere [35]. Three-dimensional structures of cutin and suberin macromolecules are not well understood, since their constitutive moieties can vary appreciably both in specific nature and relative abundance within macromolecular network [30]. However, Kolattukudy [36] suggested hypothetical models of cutin and suberin structures as shown in Figures 1 and 2 , respectively.

In this paper, we present results of electrical switching properties of thin films of NFSC as a function of annealing temperature, irradiation with laser beam, and poling temperature. Fourier transform infrared (FT-IR) spectroscopy of NFSC is first analyzed and compared with FT-IR of cutin and suberin. Possible composition of monomers in NFSC is inferred from this comparison. Current-voltage $(I-V)$ characteristics of NFSC under different conditions are analyzed and finally a discussion of possible electrical switching mechanism is presented.

\section{Experimental}

2.1. Sample Preparation. FT-IR spectrometer of Hyper model 8400 series was used to obtain IR spectra of pristine and annealed samples. Cuticle samples cut out from a seed (Figure 3) were fitted into the sample holder of the FT-IR spectrometer.
Annealing was done by placing the sample inside a temperature-controlled furnace that was fitted inside an electrically shielded cage of Lindberg/Blue Tube Furnace TF55035C. Samples were annealed at various temperatures of $320 \mathrm{~K}, 350 \mathrm{~K}$, and $400 \mathrm{~K}$ for 30 minutes each. A mechanical mask was made by cutting circular holes of diameter $0.56 \mathrm{~cm}$ through a thin soft cardboard of dimensions $3 \mathrm{~cm} \times 8 \mathrm{~cm}$. Samples were mounted on each of the holes and then irradiated with $\mathrm{He}-\mathrm{Ne}$ laser beam of wavelength $632.8 \mathrm{~nm}$ in a dark room. Irradiation was done separately on different samples for a time period of 10 minutes, 30 minutes, or 60 minutes each. Electrode coating on the film of pristine, preannealed, and preirradiated samples was done by using quick drying and highly conducting flash-dry silver paint obtained from SPI Supplies, Pa, USA. A mask of circular aperture of $0.56 \mathrm{~cm}$ diameter was used while coating to ensure uniformity in sizes of the coated regions. Circular aluminum foils of the same diameter were placed on the coated surfaces such that samples were sandwiched between two aluminum electrodes. The same flash-dry silver paint was used to connect thin wires onto the aluminum electrodes. A sample holder is shown in Figure 4. The thickness of the films was measured by a micrometer screw gauge and found to be $4 \pm 0.02 \mu \mathrm{m}$.

2.2. Current-Voltage (I-V) Measurement. Before measuring voltage and current, the samples in Figure 4 were electrically 


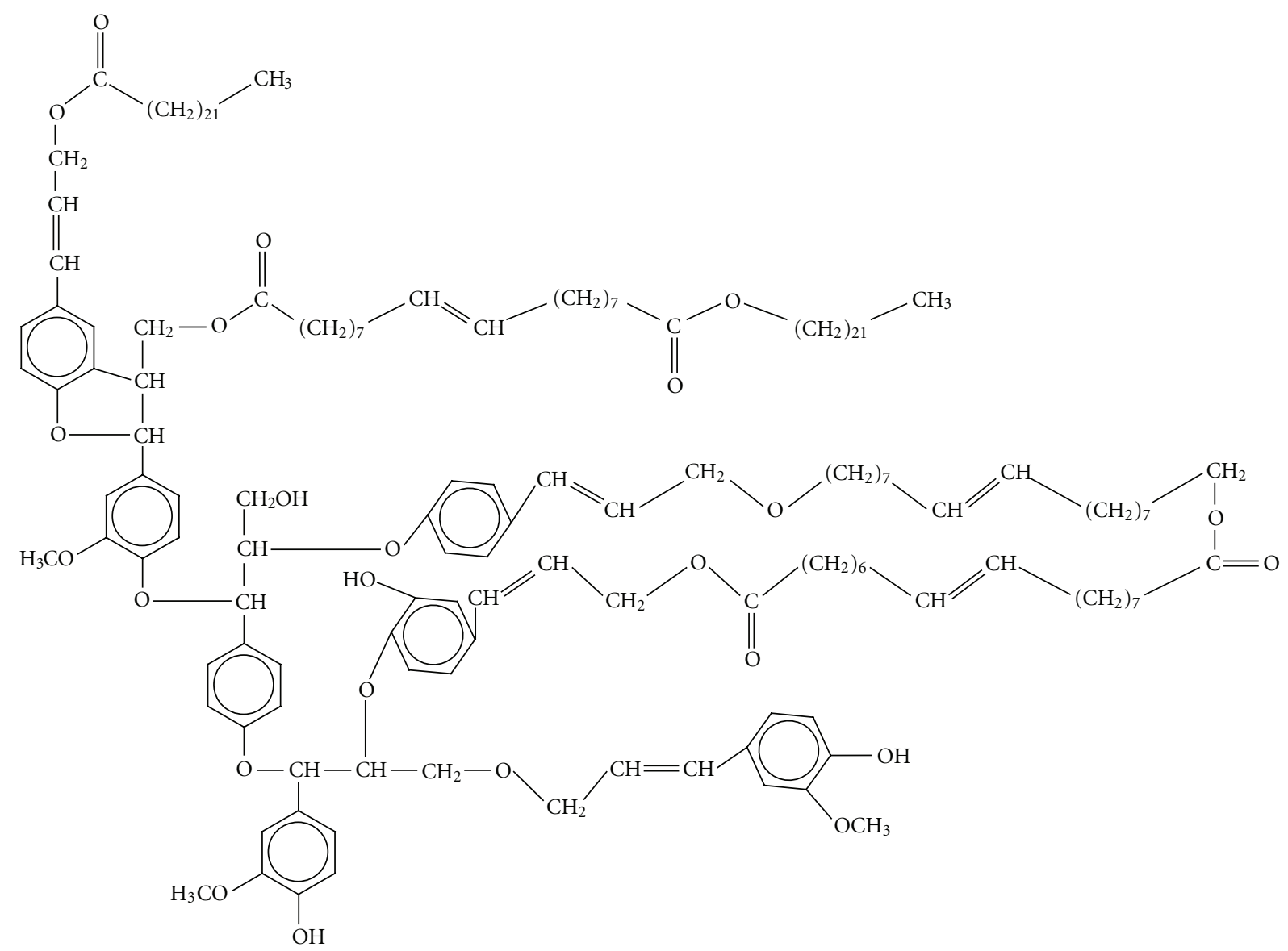

FIgURE 2: Hypothetical structure of suberin polymer.

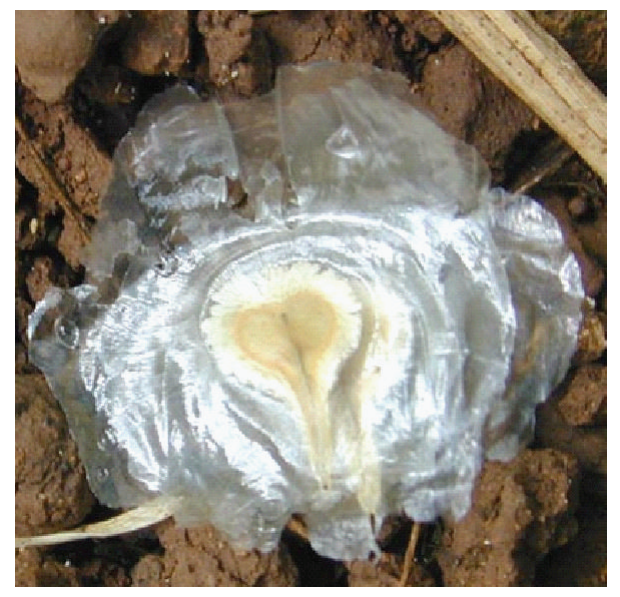

FIgURE 3: Thin and transparent cuticle attached to the Nandi flame seed.

annealed by driving high current through them. This was done by driving current of $0.3 \mathrm{~A}$ through the samples for 10 15 minutes. This reduced contact resistance and improved ohmic properties of the contact. Resistance of the contact was found to decrease considerably after it had been electrically annealed. $I-V$ for both annealed and irradiated samples
NFSC sample

sandwiched between $\mathrm{Al}$ electrodes

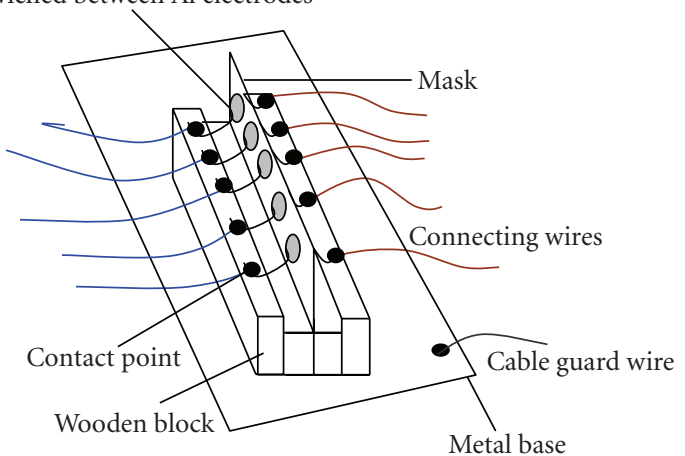

FIGURE 4: Sample holder. Thin wires are connected on both sides of the samples.

was measured using Keithley 2400 source meter. The setup of the sample holder shown in Figure 4 was shielded with aluminum metal cage coupled with an electrical insulating material in order to minimize disturbances from electrical noise, temperature fluctuation, and electromagnetic waves. $I-V$ data for each treated sample was determined with 
cable guard on. The cable guard of the source meter was activated in order to minimize leakage current. Step voltages of $0.1 \mathrm{~V}$ (within a range of $0.1 \mathrm{~V}-1.0 \mathrm{~V}$ ), $1.0 \mathrm{~V}$ (within a range of $1.0 \mathrm{~V}-10.0 \mathrm{~V}$ ), and $5.0 \mathrm{~V}$ (within a range of $10.0 \mathrm{~V}-40.0 \mathrm{~V}$ ) were sourced for both forward and reverse biases. When measuring current at poling temperature, a sample sandwiched between aluminum electrodes was placed inside the Lindbarg/Blue Tube. Furnace temperature was set to a constant value while recording current-voltage measurements.

\section{Results and Discussion}

Figure 5 shows FT-IR spectrum of pristine NFSC. IR absorption bands of NFSC are compared with IR bands of cutin and suberin biopolyesters found in plant cuticles. IR bands and band assignments are listed in Table 1. Cutin and suberin IR data bands and comments in Table 1 are based on literature $[33,37-39]$. The IR spectrum of NFSC shows a wide band at $3348 \mathrm{~cm}^{-1}$ assigned to $\mathrm{O}-\mathrm{H}$ stretching vibration. This band is correlated to absorptions at $3300 \mathrm{~cm}^{-1}$ and $3464 \mathrm{~cm}^{-1}$ in cutin and subrin, respectively, and is caused by presence of alcoholic and phenolic hydroxyl groups involved in hydrogen bonds [40]. Bands located near $2900 \mathrm{~cm}^{-1}$ and $2300 \mathrm{~cm}^{-1}$ resulted from $\mathrm{C}-\mathrm{H}$ asymmetric and symmetric stretching vibrations of methoxyl and methylene groups, respectively $[37,39]$. Methylene is the most repeated structural unit in the cutin biopolyester [37]. Around $1750-1630 \mathrm{~cm}^{-1}$ wavenumbers, absorption bands are caused by stretching vibrations of carbonyl groups. Absorption around $1603 \mathrm{~cm}^{-1}$ and $1513 \mathrm{~cm}^{-1}$ is assigned to the stretching of $\mathrm{C}=\mathrm{C}$ bonds and the stretching of benzenoid rings. Absorption bands in the range $1300-1210 \mathrm{~cm}^{-1}$ are related to asymmetric vibration of $\mathrm{C}-\mathrm{O}-\mathrm{C}$ linkages in ester to esters or phenolic groups. Other IR absorption band assignments of NFSC in comparison with those of cutin and suberin are summarized in Table 1. IR bands of NFSC between $3300-1500 \mathrm{~cm}^{-1}$ result from components of both cutin and suberin while bands around $1400-1200 \mathrm{~cm}^{-1}$ are more related to those of suberin components as shown in Table 1. Types of monomers in cutin and suberin are shown in Table 2. Figure 6 shows FTIR spectrum of pristine NFSC in comparison with spectrum of NFSC sample annealed at $400 \mathrm{~K}$. The spectra show that IR absorption peaks for pristine and annealed samples occurred at nearly the same frequency. This means that annealing $(400 \mathrm{~K})$ does not destroy the functional groups in the sample.

Figure 7(a) shows $I-V$ characteristics of pristine and annealed samples. $I-V$ curves were drawn as log-log plots because in many cases drastic conductivity changes of several orders of magnitude were observed. It is noted that at certain threshold voltage, $V_{\text {th }}$ current rises rapidly by an order of about $10^{4}$. There are two distinct regions for forward bias regime. High-impedance region, which occurs before $V_{\text {th }}$, is referred to as OFF state and low-impedance region, which occurs after $V_{\text {th }}$, is known as ON state. At low voltages, $\log I$ versus $\log V$ plots are approximately linear with a slope of 1 , while at higher voltages, above a well-defined threshold voltage $V_{\text {th }}$, the plots are again approximately

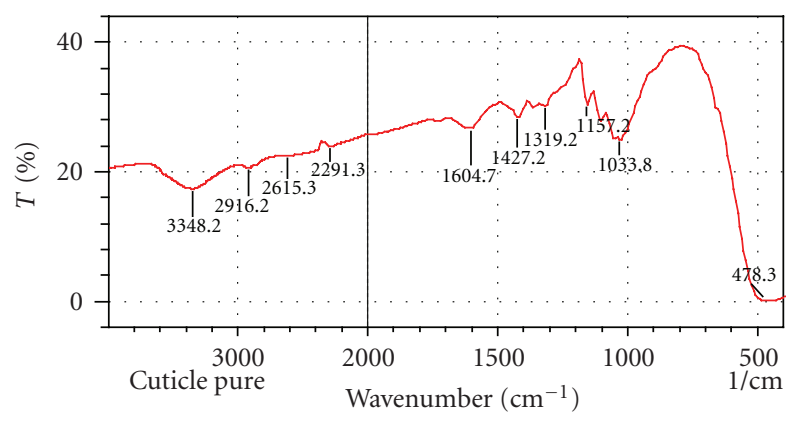

FIGURE 5: FT-IR spectrum of pristine NFSC.

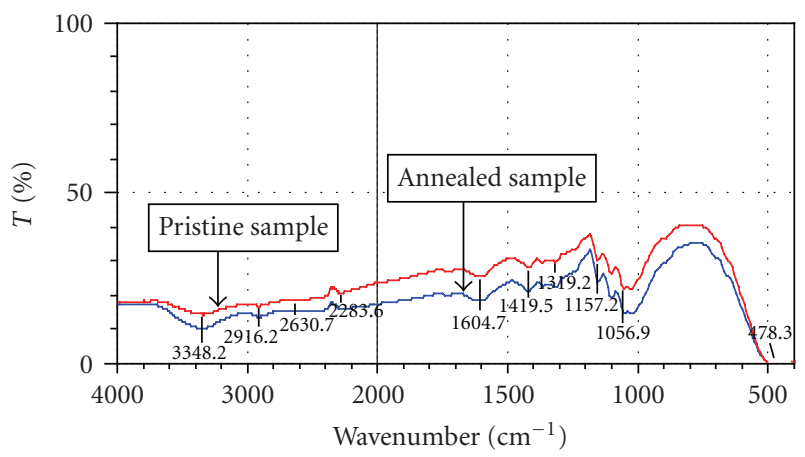

Figure 6: Comparison between FT-IR spectra of pristine and annealed $(400 \mathrm{~K})$ sample.

linear with a slope of $1.8 \pm 0.2$. The plots show that at low voltages, OFF state, current follows ohms law but after switching to ON state at higher voltages, current follows a power law dependence given by $I=V^{n}$, where $n=$ $1.8 \pm 0.2$ obtained from the slopes. This shows that the $\mathrm{ON}$-state region is governed by space charge limited current (SCLC) controlled by single trapping level, the injecting carrier concentration dominating the thermally generated carriers. Current increased by an order of $10^{2}$ after switching to $\mathrm{ON}$ state. It was also noted that current did not follow the same path on decreasing applied electric field, hence indicating that the samples exhibit electrical switching with memory. This hysteresis effect was not erased by annealing or irradiating the samples. The threshold voltage $V_{\text {th }}$ for pristine samples was $5 \pm 0.5$ volts which is higher than $V_{\text {th }}$ reported for some synthetic polymers; polymethylmethacrylate $(1.6 \mathrm{~V})$, polystyrene $(4.5 \mathrm{~V})$ [12], phthalocyanine $(0.3 \mathrm{~V})$ [41], 2,6(2,2-bicyanovinyl) pyride $(5.01 \mathrm{~V})$ [42], Langmuir-Blodgett $(1.0 \mathrm{~V})$ [43]. The width of $V_{\text {th }}$ or transition voltage during switching from $\mathrm{OFF}$ to $\mathrm{ON}$ states was about 1.0 V. All samples returned to OFF state after about 30 minutes. Figure 7(b) shows that $V_{\text {th }}$ increased nonuniformly from $5-9 \mathrm{~V}$, with increase in annealing temperature. A decrease in magnitude of the negative dielectric anisotropy during annealing is a major reason for the increase in $V_{\text {th }}$ for the annealed samples [41].

Figure 8(a) shows that irradiated samples have similar $I-V$ switching properties as annealed samples. The curves show electrical switching and memory effect with $V_{\text {th }}$ that 
TABLE 1: FT-IR frenquencies $\left(\mathrm{cm}^{-1}\right)$ of cutin, suberin, and NFSC. * Benítez et al. [37], Cordeiro et al. [39].

\begin{tabular}{|c|c|c|c|c|}
\hline Cutin* & Suberin* & NFSC & Band assignment & Comments* \\
\hline 3300 & 3464 & 3348 & $v(\mathrm{O}-\mathrm{H})$ & Alcoholics and Phenolic hydroxyl groups \\
\hline 2924 & 2919 & 2916 & $v_{\text {ass }}(\mathrm{C}-\mathrm{H})$ & Aliphatic stretch, \\
\hline \multirow[t]{2}{*}{2852} & 2851 & & & Methoxyl groups, \\
\hline & & 2615 & $\nu_{\mathrm{s}}(\mathrm{C}-\mathrm{H})$ & Methylene groups \\
\hline 1736 & 1738 & 1682 & $v_{\text {as }}\left(\mathrm{COO}^{-}\right)$ & Ester carbonyl, \\
\hline 1731 & & & $\nu(\mathrm{C}=\mathrm{O})$ & Ketone groups \\
\hline 1630 & 1635 & & & \\
\hline \multirow{8}{*}{1530} & & 1603 & $\nu(\mathrm{C}=\mathrm{C})$ & Conjugated double bonds, \\
\hline & 1513 & & & benzenoid rings \\
\hline & 1463 & & $\delta(\mathrm{C}-\mathrm{H})$ & Aromatic ring \\
\hline & 1437 & 1427 & $\nu_{\text {ass }}(\mathrm{C}-\mathrm{H})$ & \\
\hline & 1364 & & & Metoxyl groups \\
\hline & 1300 & 1319 & & Esters linkages, \\
\hline & 1210 & & $\nu_{\text {ass }}(\mathrm{C}-\mathrm{O}-\mathrm{C})$ & Phenolics, \\
\hline & & & & Glycosidic link. \\
\hline 1167 & 1130 & 1157 & $\nu(\mathrm{C}-\mathrm{O})$ & Aliphatic alcohols or Aliphatic esters \\
\hline 1100 & & & $\nu(\mathrm{C}-\mathrm{H})$ & Benzene ring \\
\hline 1055 & 1050 & 1033 & $\begin{array}{c}\delta(\mathrm{O}-\mathrm{H}) \\
\nu(\mathrm{C}-\mathrm{O})\end{array}$ & Primary and secondary alcohols \\
\hline
\end{tabular}

increased (from 5 to $8 \mathrm{~V}$ ) with the increase in irradiation time. It is also noted that irradiation increased electrical conductivity especially in the reverse bias. It also increased the gap between OFF state and ON state currents in the forward bias. Increase in conductivity for irradiated samples can be attributed to dissociation of primary valence bonds into radicals. The dissociation of $\mathrm{C}-\mathrm{C}$ and $\mathrm{C}-\mathrm{H}$ bonds leads to degradation and cross-linking which improves electrical conductivity [44]. Exposure of polymers to ionizing radiation produces current carriers in terms of electron and holes which may be trapped in the polymer matrix at low temperatures [45]. If the original conductivity is small, then the presence of these carriers produces an observable increase in the background conductivity of the polymer. Irradiation of polymers also results in excitations of its molecules and creation of free electrons and ions that migrate through the polymer network till they are trapped. The electronic and ionic configurations created cause changes in the electric conductivity. Figure 8(b) shows that $V_{\text {th }}$ increased almost linearly with the increase in irradiation time. Since irradiating time is associated with an increase in irradiation dose [46], increasing duration of irradiation could have led to an increase in the number of chain ends acting as trapping sites which increased $V_{\text {th }}$.

Figure 9 shows $I-V$ curves of NFSC obtained at different poling/measurement temperature. These curves show that electrical current increases with the increase in measurement temperature. This is due to thermal excitation of the trapped charges across the potential barrier. The curves also show that forward bias characteristics for $320 \mathrm{~K}$ and $350 \mathrm{~K}$ have two distinct regions, the ON and OFF states which are typical of electrical switching process. It is also noted that $V_{\text {th }}$ decreases with the increase in measurement temperature and that switching and memory effects almost disappeared at higher temperatures $(370 \mathrm{~K})$. Increased temperature facilitates diffusion of ions in the space charge polarization. Thermal energy may also aid in overcoming activation barrier for orientation of polar molecules in the direction of the field [47].

Although several examples of conductance switching have been investigated extensively, switching mechanism in most cases is unknown and is the subject of some controversy [5]. Several possible mechanisms for the conductance transition observed in NFSC were considered. This resulted in the identification of a possible mechanism that is in agreement with the available electrical conductance data and possible structure of this system. The first mechanism considered was the quantum interference of different propagation parts within the molecules which involve permutation of highest occupied molecular orbitals (HOMOs) and lowest unoccupied molecular orbitals (LUMOs) [48].

This can be explained by considering the potential barrier distinction between the metal contact regions. Under the positive bias, the electrons are easier to inject from the electrode into the molecule and are more difficult to transfer from the molecule to the opposite electrode, thus resulting in the charging of the molecule. In this bias, charging (charging energy) in the molecule moves the HOMO up with respect to Fermi level, whereas under negative bias, the deficit of electrons in the molecule moves the HOMO down [49]. Consequently, it can be reasonably concluded that a smaller (larger) positive (negative) bias is necessary to drive the HOMO into the HOMO-LUMO gap, hence allowing large currents to flow. This could also result from permutation of frontier orbitals, $\mathrm{HOMO}_{-1}$, and $\mathrm{LUMO}_{+1}$. In the absence of electric field, $\mathrm{HOMO}_{-1}$ is localized on the acceptor group 
TABLE 2: Types of monomers and their \% abundance in cutin and suberin biopolyesters. Pollard et al. [30].

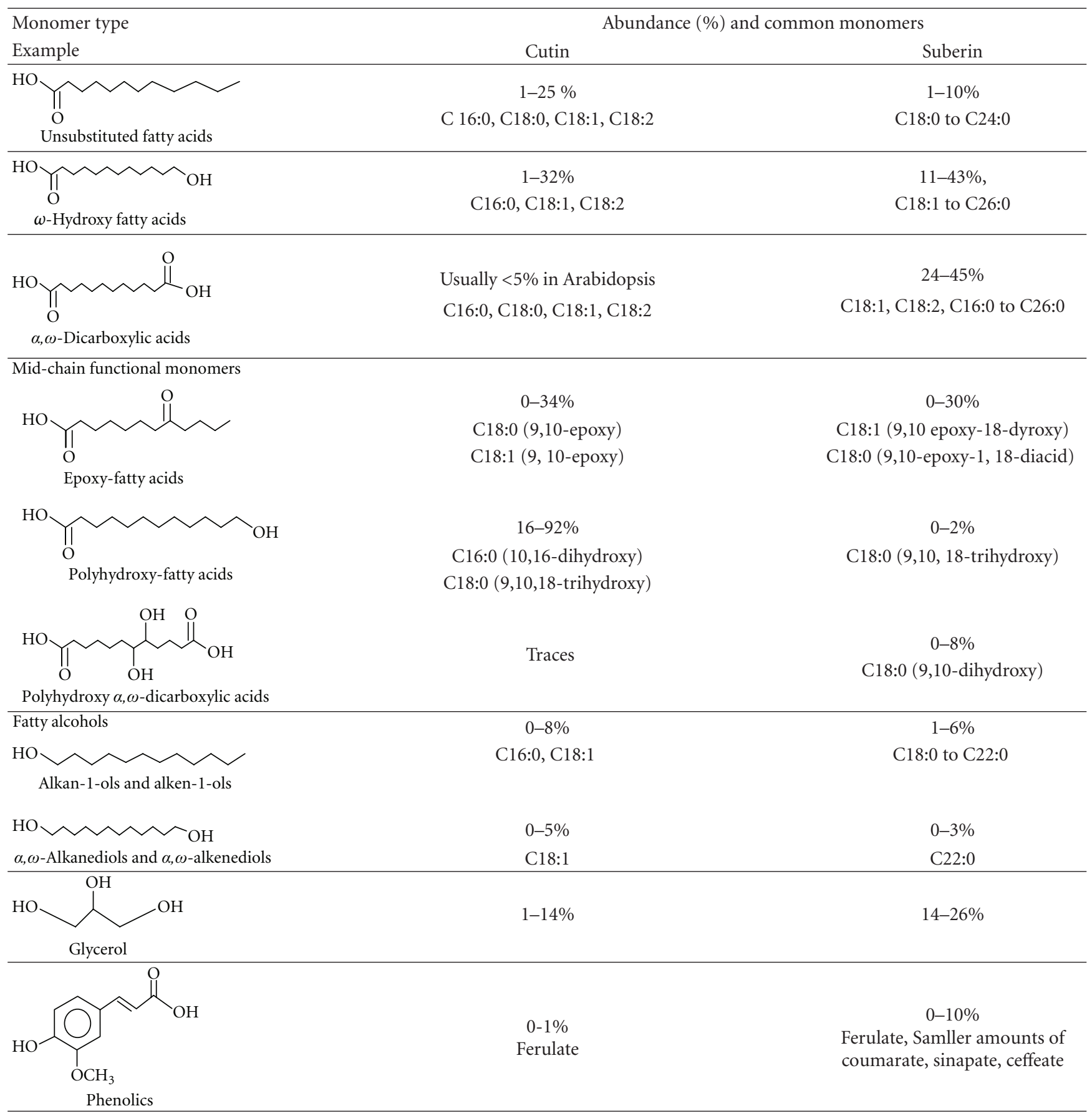

and $\mathrm{LUMO}_{+1}$ on the donor group. When the external field is applied, electron orbitals are "pulled" toward the acceptor group reducing the HOMO-LUMO gap of frontier orbitals and the switching and hybridization between $\mathrm{HOMO}_{-1}$ and $\mathrm{LUMO}_{+1}$ take place. As the strength of the field increases, the HOMO-LUMO gap in the molecular spectrum becomes smaller and the HOMO, $\mathrm{HOMO}_{-1}$, and LUMO orbitals split into the HOMO-LUMO gap, and hence become delocalized. Figure 10 shows a schematic diagram of the switching effect under an electric field. However, this explanation does not account for the formation of hysteresis and the return of the samples to OFF state within 30 minutes.

Another possible mechanism considered for electrical switching in NFSC is nondifferential resistance (NDR) which results from the injection of electrons from electrodes leading to build up of space charges across the metal electrodes and formation of high-field domains (or an accumulation layer due to dipole formation) $[49,50]$. Formation of highfield regions leads to elongation of low-field regions till the interface separating the high- and low-field regions lies on 


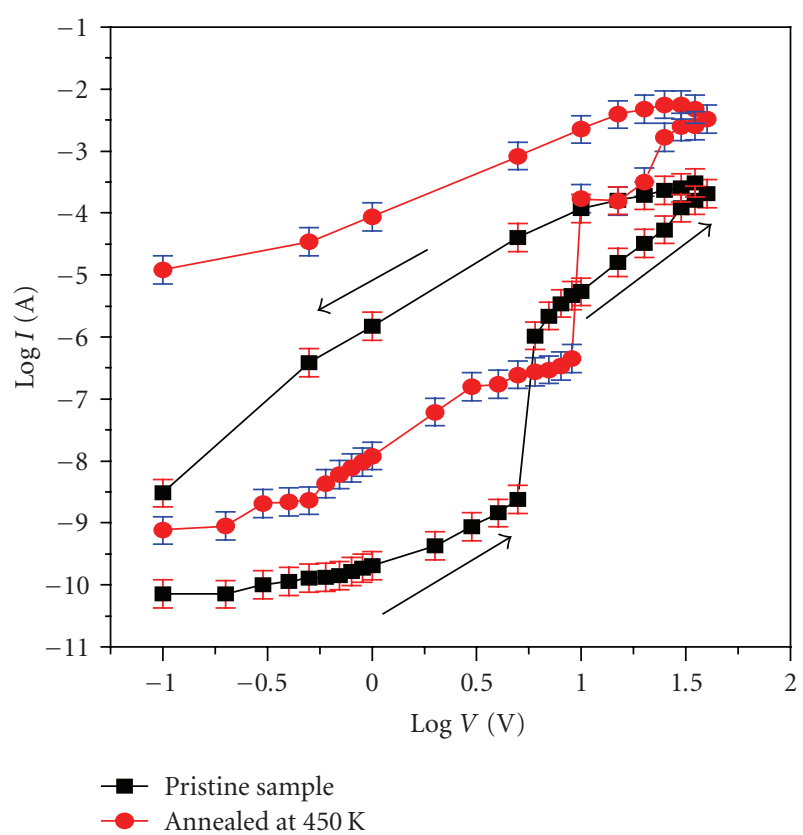

(a)

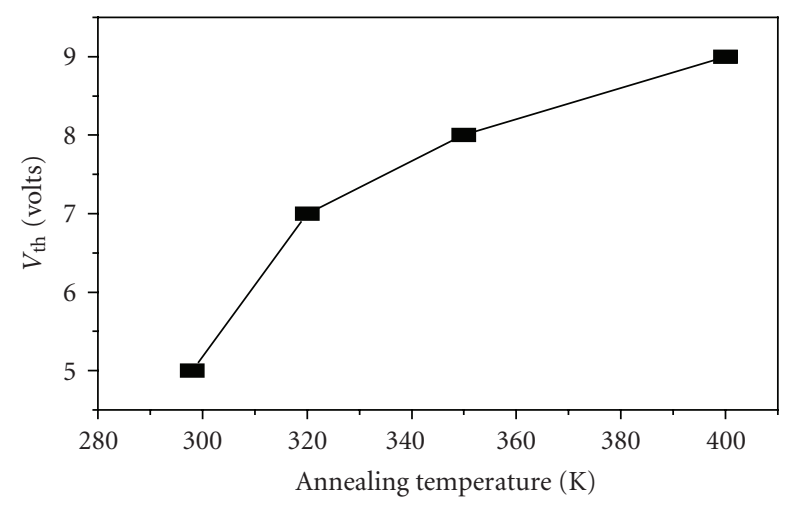

(b)

FIgURE 7: (a) I-V Switching characteristics for pristine and annealed samples. (b) Variation of threshold voltage $V_{\text {th }}$ with annealing temperature.

the same potential and is perpendicular to the current flow direction [51]. Electron injection into molecules sandwiched between metal electrodes was originally proposed to explain NDR [50] but was criticized due to the requirement for a counterion to neutralize the resulting space charge. The mechanism would also generate a negative space charge in the molecules, which cannot be compensated easily. As more charge is injected, space charge potential will increase, and the voltage required for charge injection also increases. While space charge effects may not be favorable for electrical switching, they do not appear to prevent switching a minority of molecules in the absence of counterions [5].

The most likely source of conductance switching in NFSC is the formation of quinoid and semiquinone structures from phenolic compounds present in plant cuticles $[52,53]$

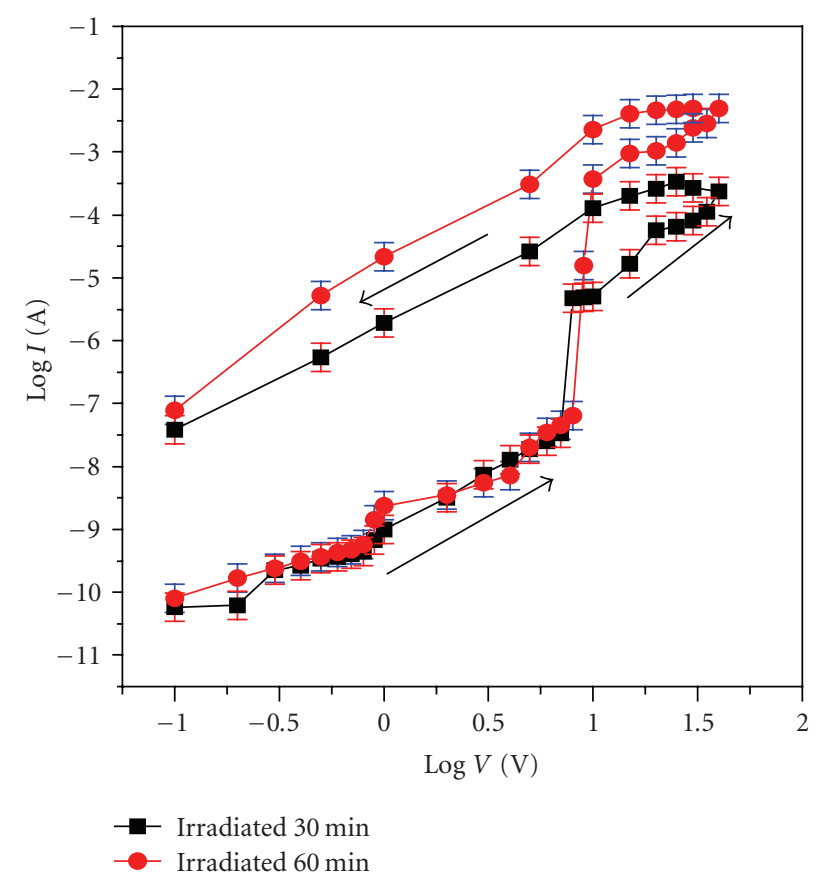

(a)

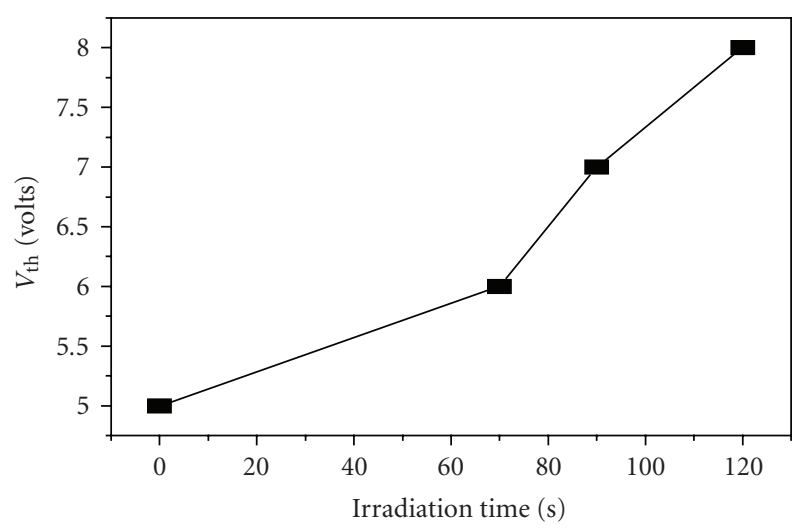

(b)

FIGURE 8: (a) $I-V$ switching characteristics for irradiated samples. (b) Variation of threshold voltage $V_{\text {th }}$ with irradiation time.

and which are presumably present in NFSC. It has been observed that electrical switching molecules are likely to have one or more phenyl rings and hemiquinone structures $[5,54]$. Organic conductors are assemblies of molecules whose HOMO orbitals are partially filled [55]. This allows for easy proton-electron transfer within molecules which incidentally may cause conductance switching. Aviram et al. [54] suggested that electron-proton motion within hemiquinones molecules that comprised of catechol and oquinone, molecules between two contacts may switch the molecules to low-impedance $(\mathrm{ON})$ state due to the formation of semiquinones-free radicals. When an electron is injected into the molecules from the metal contact, it is gained by an electron acceptor (o-quinone) molecule. An electron donor (catechol) molecule then transfers the electron to 


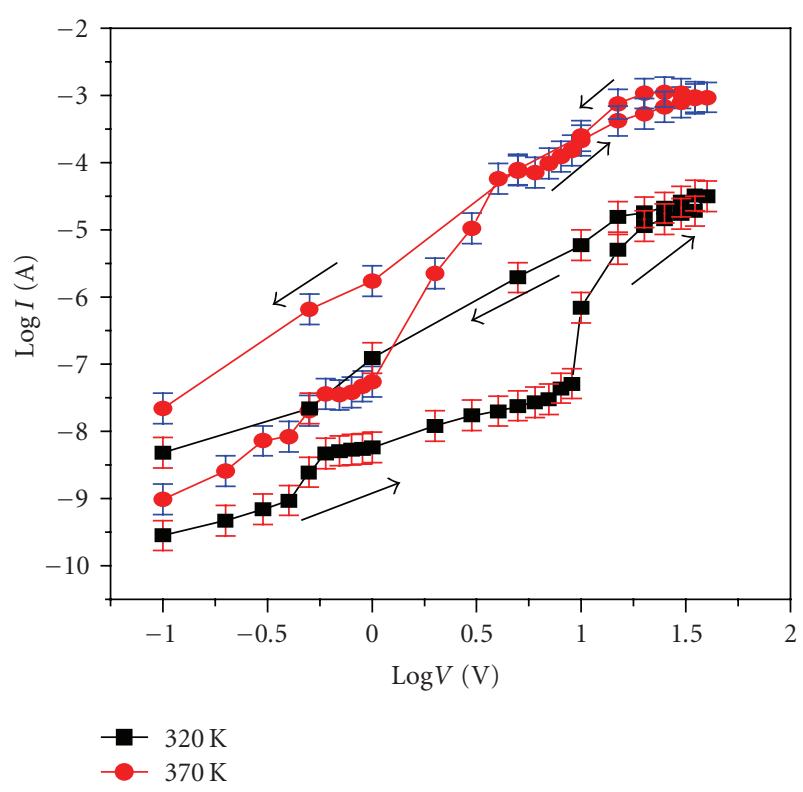

FIGURE 9: $I-V$ switching characteristics at different poling temperatures.

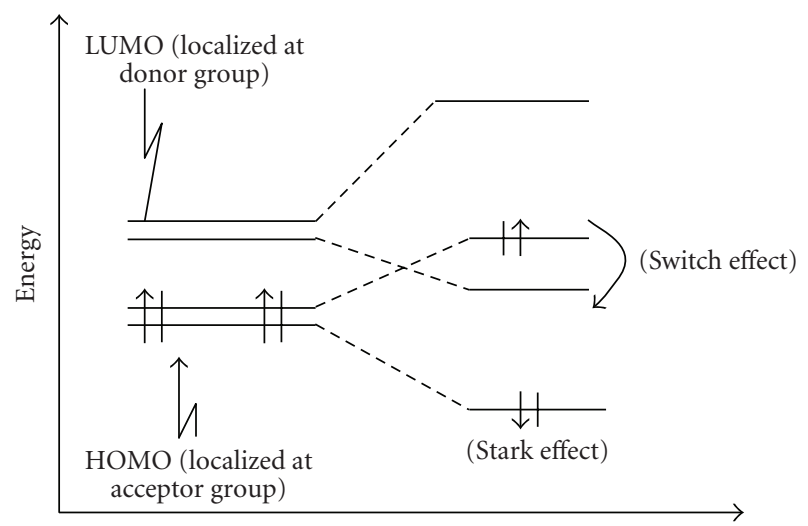

Figure 10: Schematic diagram for the switching effect under an electric field.

the opposite contact thus allowing flow of charges. The dipole, thus, setup would be rapidly cancelled by oneproton motion from the positively charged catechol to the negatively charged quinone group, giving rise to two neutral semiquinones which are free radicals responsible for the onset of large current. The threshold switching occurs when the fields become sufficient for proton motion and level matching for the electron motion. Since conduction in cutin involves protons [56], conductance switching in NFSC is feasible under this mechanism. However, hysteresis effect is better explained by considering the formation of quinoid structures. Ferulic acids, which are compounds of phenolic nature with some ionizable caboxylic and hydroxyl groups, can act as a charge network forming pathways for electronproton transfer. An electron transfer into a phenolic compound may result in the formation of quinoid structure with many resonance forms. The quinoid form is planar, and is highly conjugated compared to phenyl groups. As the electric field increases, quinoid structures become delocalized over the entire structure. This is the basis for the change of conductance state from OFF to $\mathrm{ON}$ state. In addition to increased conjugation and planarity, a decrease in HOMOLUMO gap in the quinoid structures occurs in all forms of "switching" molecules [5]. Benzene ring rotation and bond length changes during the formation of quinoid result in activation barriers which are presumably the origin of the temperature dependence conductance. Activation barrier associated with the structural rearrangement is responsible not only for the activation barrier but also for the switching hysteresis. The quinoid form is apparently the higher energy form in the absence of an electric field, but is the favored form in a high field [5]. Reorganization energy is sufficiently large that the quinoid form survives after the field is removed, at least for several minutes to several hours [5]. High conductance high field of the reverse regime may also be due NDR and Fowler-Nordheim field emission process which involves tunneling of free carriers in the bulk of the material [56].

\section{Conclusion}

$I-V$ characteristics of Nandi flame seed cuticle biomaterial have shown reproducible switching and memory properties. The switching and memory effects are explained in terms of formation of semiquinones and quinoids structures from phenolic compounds due to redox processes. The switched samples lost memory within 10-30 minutes. Threshold voltage $V_{\text {th }}$ for pristine samples was about $5 \mathrm{~V}$. $V_{\text {th }}$ increased to about $6-8 \mathrm{~V}$ after irradiation with laser light of wavelength $632.8 \mathrm{~nm}$. Annealing also increased $V_{\text {th }}$ by the same magnitude. Hysteresis effect in the $I-V$ curves is due to the presence of quinoid radicals. At a poling temperature of $370 \mathrm{~K}$, both switching and memory effects almost disappeared. Current in the OFF state was ohmic but after switching to $\mathrm{ON}$ state, current was governed by space charge-limited current (SCLC). These results have shown that NFSC has electrically switchable molecules which can be explored further for their application in gas sensing and electrical switching circuits.

\section{Acknowledgments}

The authors wish to thank the chairman of the Chemistry Department, Jomo Kenyatta University, Kenya, for providing FT-IR equipment. A useful discussion with Dr. Periklis Papadopolous of University of Leipzig, Germany, concerning FT-IR analysis is gratefully acknowledged.

\section{References}

[1] M. A. Reed, J. Chen, A. M. Rawlett, D. W. Price, and J. M. Tour, "Molecular random access memory cell," Applied Physics Letters, vol. 78, no. 23, pp. 3735-3737, 2001.

[2] H. Qu, W. Yao, T. Garcia, et al., "Nanoscale polarization manipulation and conductance switching in ultrathin films of a ferroelectric copolymer," Applied Physics Letters, vol. 82, no. 24, pp. 4322-4324, 2003. 
[3] H. J. Gao, K. Sohlberg, Z. Q. Xue, et al., "Reversible, nanometer-scale conductance transitions in an organic complex," Physical Review Letters, vol. 84, no. 8, pp. 1780-1783, 2000.

[4] A. Aviram and M. A. Ratner, "Molecular rectifiers," Chemical Physics Letters, vol. 29, no. 2, pp. 277-283, 1974.

[5] A. O. Solak, S. Ranganathan, T. Itoh, and R. L. McCreery, "A mechanism for conductance switching in carbon-based molecular electronic junctions," Electrochemical and SolidState Letters, vol. 5, no. 8, pp. E43-E46, 2002.

[6] C. P. Collier, E. W. Wong, M. Belohradský, et al., "Electronically configurable molecular-based logic gates," Science, vol. 285, no. 5426, pp. 391-394, 1999.

[7] C. P. Collier, G. Mattersteig, E. W. Wong, et al., "A [2] catenanebased solid state electronically reconfigurable switch," Science, vol. 289, no. 5482, pp. 1172-1175, 2000.

[8] I. D. Parker, "Carrier tunneling and device characteristics in polymer light-emitting diodes," Journal of Applied Physics, vol. 75, no. 3, pp. 1656-1666, 1994.

[9] A. Bune, S. Ducharme, V. Fridkin, et al., "Novel switching phenomena in ferroelectric Langmuir-Blodgett films," Applied Physics Letters, vol. 67, no. 26, pp. 3975-3977, 1995.

[10] J. O. Siro, Switching behaviour in polymethymethacrylate thin films the effect of blending, doping and temperature, M.S. thesis, Kenyatta University, Nairobi, Kenya, 1997.

[11] B. Wafula and G. Katana, "Current-voltage characterization of doped polystyrene thin films with view to gas sensing," East African Journal of Physical Sciences, vol. 5, no. 1, pp. 3-9, 2004.

[12] G. Katana and A. Musyoki, "Fabrication and performance testing of gas sensors based on organic thin films," Journal of Polymer Materials, vol. 24, no. 4, pp. 387-394, 2007.

[13] D. D. Eley, N. C. Lockhart, and C. N. Richardson, "Electrical properties and structural transitions in the mitochondrion," Journal of Bioenergetics and Biomembranes, vol. 9, no. 5, pp. 289-301, 1977.

[14] K. Leszek, M. Ewa, and J. Feliks, "Changes in the electrical conductivity of the $\gamma$-irradiated BAT collagen," Polish Journal of Medical Physics and Engineering, vol. 8, no. 3, pp. 157-164, 2002.

[15] H. Mallick and A. Sakar, "An experimental investigation of electrical conductivities in biopolymers," Bulletin of Material Science, vol. 23, no. 4, pp. 319-324, 2000.

[16] H. Mallik and A. Sarkar, "Electrical characterization of ion conducting biopolymeric gel complexes," Journal of NonCrystalline Solids, vol. 352, no. 8, pp. 795-800, 2006.

[17] T. J. Lewis and P. J. Bowen, "Electronic processes in biopolymer systems," IEEE Transactions on Electrical Insulation, vol. 19, no. 3, pp. 254-256, 1984.

[18] A. Tiwari and S. P. Singh, "Synthesis and characterization of biopolymer-based electrical conducting graft copolymers," Journal of Applied Polymer Science, vol. 108, no. 2, pp. 1169$1177,2008$.

[19] J. Ramírez-Salgado, "Study of basic biopolymer as proton membrane for fuel cell systems," Electrochimica Acta, vol. 52, no. 11, pp. 3766-3778, 2007.

[20] E. M. Heckman, J. G. Grote, F. K. Hopkins, and P. P. Yaney, "Performance of an electro-optic waveguide modulator fabricated using a deoxyribonucleic-acid-based biopolymer," Applied Physics Letters, vol. 89, no. 18, Article ID 181116, 3 pages, 2006.

[21] A. Tiwari, "Synthesis and characterization of $\mathrm{pH}$ switching electrical conducting biopolymer hybrids for sensor applications," Journal of Polymer Research, vol. 15, no. 4, pp. 337-342, 2008.
[22] D. F. Rosa and P. Danny, DNA-Based Nanoelectronics, Humana Press, Totowa, NJ, USA, 2006.

[23] E. Ruiz-Hitzky, M. Darder, and P. Aranda, "Functional biopolymer nanocomposites based on layered solids," Journal of Materials Chemistry, vol. 15, no. 35-36, pp. 3650-3662, 2005.

[24] J. F. Villena, E. Domínguez, D. Stewart, and A. Heredia, "Characterization and biosynthesis of non-degradable polymers in plant cuticles," Planta, vol. 208, no. 2, pp. 181-187, 1999.

[25] K. E. Espelie, R. W. Davis, and P. E. Kolattukudy, "Composition, ultrastructure and function of the cutin- and suberincontaining layers in the leaf, fruit peel, juice-sac and inner seed coat of grapefruit (Citrus paradisi Macfed.)," Planta, vol. 149, no. 5, pp. 498-511, 1980.

[26] J. J. Benítez, R. García-Segura, and A. Heredia, "Plant biopolyester cutin: a tough way to its chemical synthesis," Biochimica et Biophysica Acta, vol. 1674, no. 1, pp. 1-3, 2004.

[27] A. Heredia, "Biophysical and biochemical characteristics of cutin, a plant barrier biopolymer," Biochimica et Biophysica Acta, vol. 1620, no. 1-3, pp. 1-7, 2003.

[28] C. Nawrath, "Unraveling the complex network of cuticular structure and function," Current Opinion in Plant Biology, vol. 9, no. 3, pp. 281-287, 2006.

[29] C. E. Jeffree, "Structure and ontogeny of plant cuticles," in Plant Cuticles: An Integrated Functional Approach, pp. 33-82, BIOS Scientific, Oxford, UK, 1996.

[30] M. Pollard, F. Beisson, Y. Li, and J. B. Ohlrogge, "Building lipid barriers: biosynthesis of cutin and suberin," Trends in Plant Science, vol. 13, no. 5, pp. 236-246, 2008.

[31] I. Molina, G. Bonaventure, J. Ohlrogge, and M. Pollard, "The lipid polyester composition of Arabidopsis thaliana and Brassica napus seeds," Phytochemistry, vol. 67, no. 23, pp. 2597-2610, 2006.

[32] M. A. Bernards, M. L. Lopez, J. Zajicek, and N. G. Lewis, "Hydroxycinnamic acid-derived polymers constitute the polyaromatic domain of suberin," The Journal of Biological Chemistry, vol. 270, no. 13, pp. 7382-7386, 1995.

[33] F. J. Ramirez, P. Luque, A. Heredia, and M. J. Bukovac, "Fourier transform IR study of enzymatically isolated tomato fruit cuticular membrane," Biopolymers, vol. 32, no. 11, pp. 1425-1429, 1992.

[34] W. K. Kipnusu, G. Katana, J. Rume, J. Tsuwi, A. Serghei, and F. Kremer, "Surface characterization and molecular dynamics of Nandi flame seed cuticle," East African Journal of Physical Sciences, vol. 8, no. 1, pp. 21-36, 2008.

[35] C. G. Casado and A. Heredia, "Specific heat determination of plant barrier lipophilic components: biological implications," Biochimica et Biophysica Acta, vol. 1511, no. 2, pp. 291-296, 2001.

[36] P. E. Kolattukudy, The Structure, Biosynthesis and Degradation of Wood Components, Plenum Press, New York, NY, USA, 1977.

[37] J. J. Benítez, A. J. Matas, and A. Heredia, "Molecular characterization of the plant biopolyester cutin by AFM and spectroscopic techniques," Journal of Structural Biology, vol. 147, no. 2, pp. 179-184, 2004.

[38] J. F. Villena, E. Domínguez, and A. Heredia, "Monitoring biopolymers present in plant cuticles by FT-IR spectroscopy," Journal of Plant Physiology, vol. 156, no. 3, pp. 419-422, 2000.

[39] N. Cordeiro, M. N. Belgacem, A. J. D. Silvestre, C. Pascoal Neto, and A. Gandini, "Cork suberin as a new source of chemicals_-part 1: isolation and chemical characterization of its composition," International Journal of Biological Macromolecules, vol. 22, no. 2, pp. 71-80, 1998. 
[40] B. Ivan, Characterization of natural and technical lignins using FTIR spectroscopy, M.S. thesis, Lulea University of Technology, Lulea, Sweden, 2008.

[41] D. Ottenbacher, K. D. Schierbaum, and W. Goepel, "Switching effects in metal/phthalocyanine/metal sandwich structures," Journal of Molecular Electronics, vol. 7, no. 2, pp. 79-84, 1991.

[42] Z. Q. Xue, M. Ouyang, K. Z. Wang, H. X. Zhang, and C. H. Huang, "Electrical switching and memory phenomena in the Ag-BDCP thin film," Thin Solid Films, vol. 288, no. 1-2, pp. 296-299, 1996.

[43] K. Sakai, H. Matsuda, H. Kawada, K. Eguchi, and T. Nakagiri, "Switching and memory phenomena in Langmuir-Blodgett films," Applied Physics Letters, vol. 53, no. 14, pp. 1274-1276, 1988.

[44] H. Ashour, M. Saad, and M. Ibrahim, "Electrical conductivity for irradiated, grafted polyethylene and grafted polyethylene with metal complex," Egyptian Journal of Solids, vol. 29, no. 2, pp. 351-362, 2006.

[45] H. Adnrew and A. Len, Handbook of Radiation Effects, John Wiley \& Sons, New York, NY, USA, 1994.

[46] J. Feinleib, J. DeNeufville, S. C. Moss, and S. R. Ovshinsky, "Rapid reversible light-induced crystallization of amorphous semiconductors," Applied Physics Letters, vol. 18, no. 6, pp. 254-257, 1971.

[47] R. V. Waghmare, N. G. Belsare, F. C. Raghuwanshi, and S. N. Shilaskar, "Study of d.c. electrical conductivity of paranitroaniline doped (1:1) polyvinylchloride and poly(methyl methacrylate) polyblends," Bulletin of Materials Science, vol. 30, no. 2, pp. 167-172, 2007.

[48] Z. Zhang, Z. Yang, J. Yuan, H. Zhang, Q. Ming, and X. Deng, "Electronic transport properties of pheny1 based molecular devices," Solid State Communications, vol. 149, no. 1-2, pp. 60 63, 2009.

[49] S. M. Sze, Physics of Semiconductor Devices, John Wiley \& Sons, New York, NY, USA, 1969.

[50] J. Chen, M. A. Reed, A. M. Rawlett, and J. M. Tour, "Large on-off ratios and negative differential resistance in a molecular electronic device," Science, vol. 286, no. 5444, pp. 1550-1552, 1999.

[51] O. R. Kefa, "Memory and threshold switching in thin film PMMA polymer," Interantional Atomic Energy Agency and United Nations Educational Science and Cultural Organiazation, IC/95/65, 1995.

[52] E. J. Holloway, The Plant Cuticle, Academic Press, New York, NY, USA, 1982.

[53] J. Benavente, A. Muñoz, and A. Heredia, "Electrokinetic parameters of ion transport across isolated pepper cuticular membranes," Journal of Membrane Science, vol. 139, no. 2, pp. 147-154, 1998.

[54] A. Aviram, C. Joachim, and M. Pomerantz, "Evidence of switching and rectification by a single molecule effected with a scanning tunneling microscope," Chemical Physics Letters, vol. 146, no. 6, pp. 490-495, 1988.

[55] J. T. Devreese, R. P. Eward, and V. E. van Doren, Highly Conducting One-Dimensional Solids, Plenum Press, New York, NY, USA, 1979.

[56] H. Vestweber, R. Sander, A. Greiner, W. Heitz, R. F. Mahrt, and H. Bässler, "Electroluminescence from polymer blends and molecularly doped polymers," Synthetic Metals, vol. 64, no. 23, pp. 141-145, 1994. 

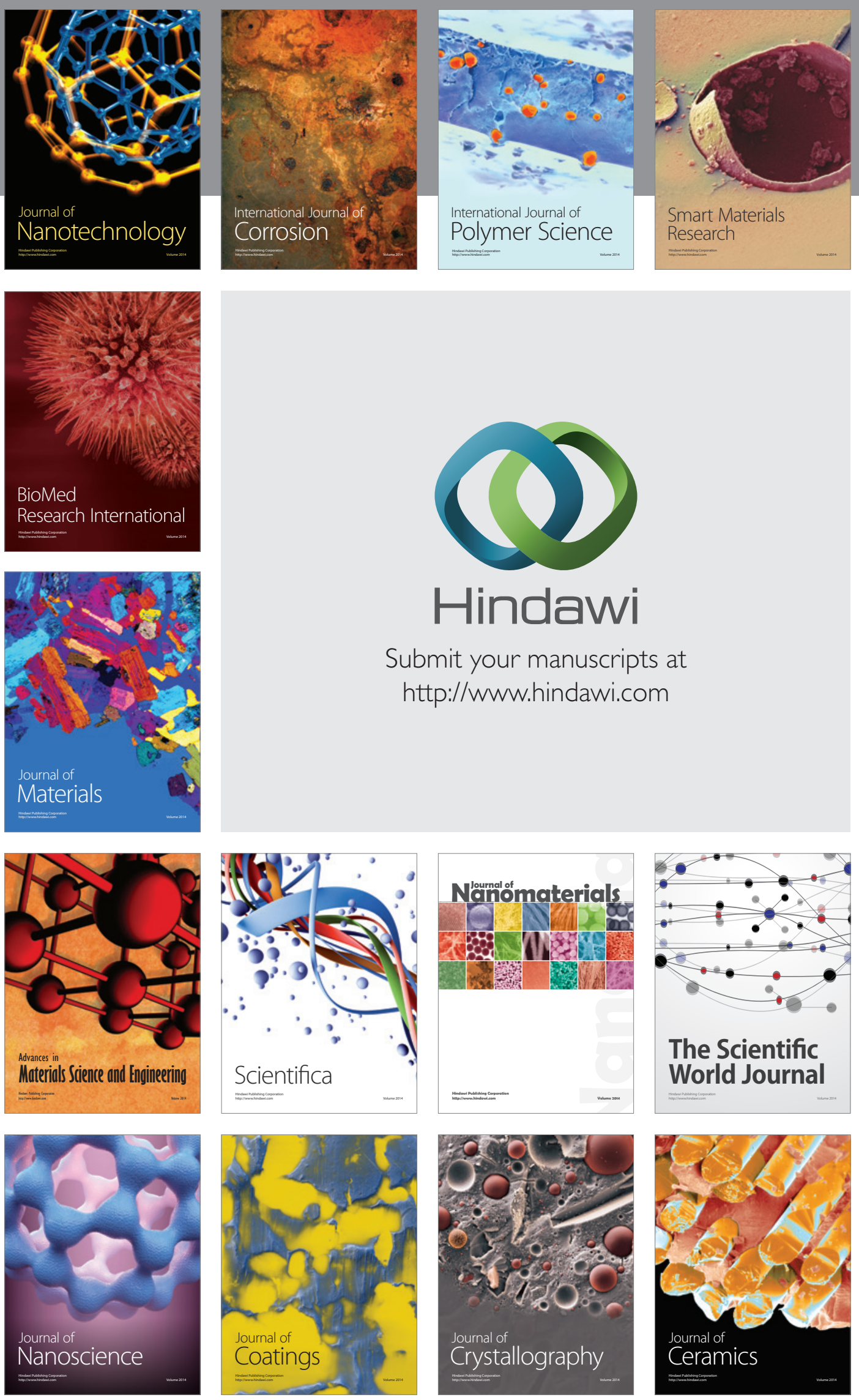

The Scientific World Journal

Submit your manuscripts at

http://www.hindawi.com

\section{World Journal}

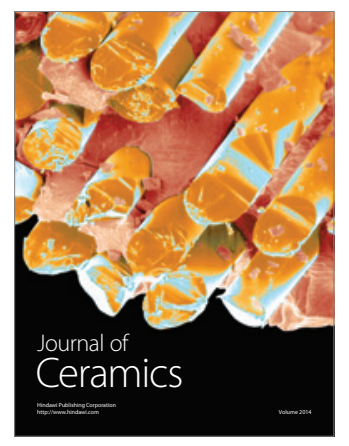

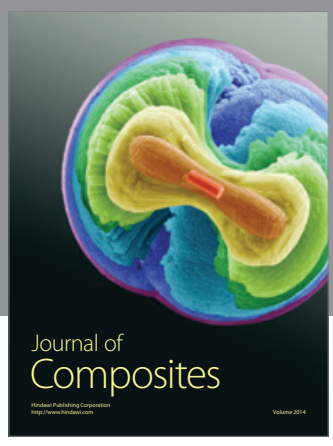
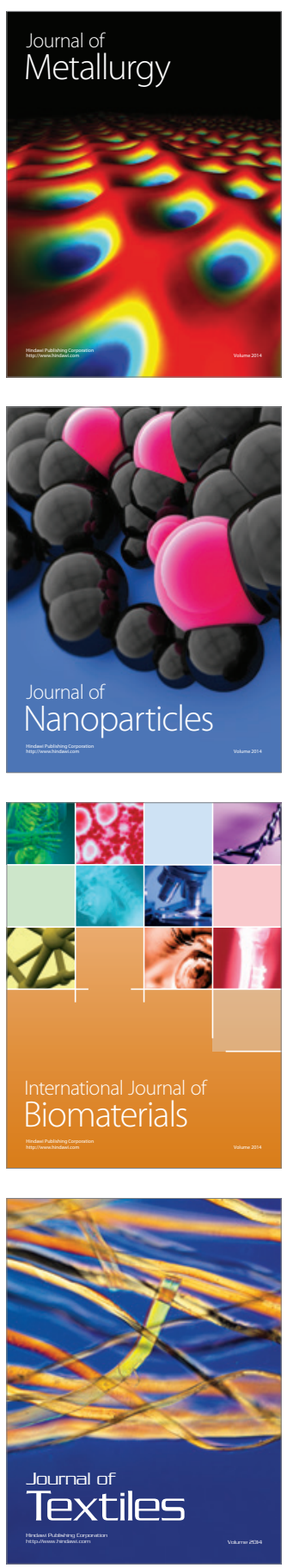